\title{
Determinación de rutas con potencial de mejora utilizando funciones de desempeño de seguridad vial: caso de Costa Rica
}

\section{Identifying dangerous routes through safety performance functions: case of Costa Rica}

\author{
Jonathan Agüero-Valverde \\ ProDus, San José, Costa Rica \\ jonathan.aguero@ucr.ac.cr
}

Fecha de recepción: 26 de julio de 2016 / Fecha de aprobación: 26 de agosto de 2016

\section{RESUMEN}

Para lograr una disminución en la frecuencia y gravedad de los accidentes de tránsito es fundamental la identificación de sitios de concentración de choques. Para tal efecto es necesario contar con datos detallados de choques y un inventario de la infraestructura vial actualizado, los cuales no se encuentran disponibles en Costa Rica. Recientemente se han popularizado modelos de choques a nivel macro que se pueden utilizar para desarrollar funciones de desempeño de seguridad vial a nivel de ruta, dado que por su nivel de agregación tienen menores requerimientos de información. De esta forma se pueden identificar rutas donde el exceso de choques sea significativo para después realizar estudios micro o detallados de sitios de concentración choques en estas rutas. Este estudio busca identificar las rutas con mayor potencial de mejora en Costa Rica utilizando funciones de desempeño de seguridad vial y el método de Bayes Empírico. Los resultados muestran que el modelo Binomial Negativo es apropiado para representar la Función de Desempeño de Seguridad Vial por ruta en Costa Rica al considerar la exposición y la sobre-dispersión. Además, el modelo indica que la ruta más peligrosa de Costa Rica en términos de exceso de muertes en carretera es la Ruta 32, seguida de la Ruta 2, la Ruta 4, la Ruta 1 y en quinto lugar Ruta 34. Las otras cinco rutas que completan las 10 más peligrosas son en orden la Ruta 35, la Ruta 21, la Ruta 36, la Ruta 27 y la Ruta 6.

PALABRAS CLAVE: Seguridad Vial, función de desempeño, Bayes empírico, rutas de concentración de choques, exceso de frecuencia de choques

\begin{abstract}
Road network screening is fundamental to reduce the frequency and severity of road crashes. For this purpose it is necessary to have a detailed crash database and an updated road inventory, which are not available in Costa Rica. Recently, macro-level crash models have become popular, since their level of aggregation have lower information requirements; they can be used to develop Safety Performance Functions (SPF) at route level. Using these SPFs routes with excess crash frequency can be identified and a detail safety screening can be performed on those routes. This study seeks to identify the most dangerous routes in Costa Rica using the SPFs and the Empirical Bayes method. The results show that the Negative Binomial model is appropriate to represent the SPFs for routes in Costa Rica since it considers the exposure and over-dispersion present in the data. In addition, the model indicates that the most dangerous routes in Costa Rica in terms of excess deaths are the Route 32, followed by Route 2, Route 4, Route 1 and Route 34. The other 5 routes completing the 10 most dangerous are in order Route 35, Route 21, Route 36, Route 27 and Route 6.
\end{abstract}

KEYWORDS: Safety Performance Function, empirical Bayes, routes with promise, excess expected crash frequency 


\section{INTRODUCCIÓN}

Los choques viales representan la principal causa de muerte prevenible en el mundo y la primera causa de muerte para personas entre 15 y 29 años de edad (Toroyan, 2015). En Costa Rica la situación es similar, solo en el 2013 los accidentes de tránsito cobraron la vida de 605 personas, lo que los sitúa como la décima causa de muerte en el país y la primera causa de muerte violenta. Además los accidentes de tránsito fueron la primera causa de muerte para personas entre los 0 y los 45 años en ese mismo año (INEC, 2014).

Los accidentes de tránsito son el resultado de una falla en el complejo sistema de interacciones entre el usuario, el vehículo y el medioambiente. Este último ámbito es donde la ingeniería de transportes puede dar su mayor contribución, particularmente en la reducción de choques a través del estudio de la interacción entre el usuario y la carretera (Evans, 2004). Dado que la mayoría de los choques viales ocurren por errores humanos (Shinar, 1978), las medidas de ingeniería en seguridad vial actuales deben buscar mejorar la interacción entre el usuario y la carretera.

Bajo estas circunstancias, es claro que para lograr una disminución en la frecuencia y gravedad de los accidentes de tránsito es fundamental la identificación de sitios de concentración de choques. Este proceso busca la identificación y ordenamiento de sitios (segmentos de carretera o intersecciones) de una red de transporte del más probable al menos probable en presentar una reducción en la frecuencia de choques con la implementación de una medida de ingeniería para la mejora de seguridad vial (AASHTO, 2010).

Para la identificación apropiada de sitios de concentración de choques es necesario contar con datos detallados de choques y un inventario de la infraestructura vial actualizado. En Costa Rica la información pormenorizada de todos los choques no está disponible en formato digital y los inventarios de carreteras no son suficientemente detallados para realizar estudios a nivel de segmento de carretera sin antes invertir una gran cantidad de recursos en la digitalización de todos los choques y la actualización del inventario de carreteras (Castro y AgüeroValverde, 2015).

Recientemente se han popularizado modelos de choques a nivel macro con el fin de incorporar elementos de seguridad vial a la planificación del transporte (Miaou y Lord, 2003; MacNab, 2004; Aguero-Valverde y Jovanis, 2006; Quddus, 2008; Huang et al 2010; Hadayeghi et al, 2010; Siddiqui at al, 2012; Aguero-Valverde, 2013).Dichos modelos se pueden utilizar para desarrollar funciones de desempeño de seguridad vial a nivel de ruta, dado que por su nivel de agregación tienen menores requerimientos de información. De esta forma se pueden identificar rutas donde el exceso de choques sea significativo para después realizar estudios micro o detallados de sitios de concentración choques en estas rutas.

El objetivo de este estudio es identificar las rutas más peligrosas de Costa Rica utilizando funciones de desempeño de seguridad vial. Para lo anterior se utilizará el método del valor esperado de exceso de frecuencia de choques con corrección de Bayes empírico, que es ampliamente reconocido como uno de los métodos más efectivos para la clasificación de sitios de concentración de choques (AASHTO, 2010; World Road Association, 2003).

\section{Funciones de desempeño de seguridad vial y Bayes Empírico}

Para analizar el riesgo de choque al que están sujetos los diferentes usuarios del sistema de transporte se debe considerar el nivel de exposición de dicho usuario. En seguridad vial, la medida de exposición más ampliamente utilizada a nivel internacional es la cantidad de kilómetros recorridos por los usuarios (Hakkert et al, 2002). El problema fundamental es que se conoce que el riesgo de choque no aumenta linealmente con la exposición por lo que la tasa de choques no es apropiada para comparar sitios (Hauer, 1995, Qin et al, 2004). Por lo tanto, la función de desempeño de seguridad vial busca modelar esa relación no lineal entre la exposición y el riesgo de choques para sitios con características similares (Hauer, 1992; 1995).

La función de desempeño de seguridad vial permite identificar el riesgo predicho para cada sitio y de esta forma comparar el riesgo observado en un sitio particular con el riesgo predicho para ese nivel de exposición utilizando una población de referencia (un grupo de sitios con características similares al sitio de estudio). Esta diferencia entre lo observado y lo predicho es lo que se conoce como el umbral de desempeño; a mayor diferencia mayor probabilidad de observar una reducción en la frecuencia de choques con la implementación de una medida de ingeniería para la mejora de seguridad vial (AASHTO, 2010).

La regresión a la media es un problema estadístico común en el análisis de datos de choques viales (Hauer et al, 2002; AASHTO, 2010). Éste es un fenómeno estadístico en el cual datos inusualmente altos o bajos tienden a ser seguidos, en el siguiente año por ejemplo, por datos que están más cercanos a la media o promedio (Barnett et al, 2005). En los estudios de seguridad vial es común contar con pocos años de datos de choques por lo que pueden observarse datos inusualmente altos o bajos que serán precedidos de datos más cercanos a la media en el futuro pero que no han sido observados. Si utilizamos los datos observados hasta ahora estaríamos sobreestimando o subestimando la 
cantidad esperada de choques en estos sitios. El método de Bayes Empírico se ha propuesto justamente para corregir este sesgo de regresión a la media (Hauer, 1997; Hauer et al, 2002; PIARC, 2003; AASHTO, 2010).

El método Bayes empírico parte de la premisa de que la inseguridad vial de un sitio se basa no solo en la cantidad de choques observados en el sitio mismo sino además en la cantidad de accidentes observados en sitios similares, conocidos como la población de referencia (Hauer, 1992). La función de desempeño de seguridad vial nos permite estimar el riesgo de choques para esa población de referencia. Entonces, el número de choques esperado para un sitio particular se estima tomando en cuenta la cantidad de choques observada en el sitio y la cantidad predicha por la función de desempeño de seguridad vial.

En resumen, el método de exceso de frecuencia de choques esperado con ajuste de Bayes empírico por medio de la función de desempeño de seguridad tiene tres ventajas que lo hacen más preciso que otros: controla por la exposición, controla por el sesgo de regresión a la media y hace uso de un umbral de desempeño (AASHTO, 2010; World Road Association, 2003).

El resto de este artículo está organizado de la siguiente manera: la Sección 2 describe la metodología en detalle, la Sección 3 presenta los datos utilizados, la Sección 4 muestra los principales resultados del estudio y finalmente la Sección 5 presenta conclusiones y recomendaciones para estudios futuros.

\section{METODOLOGÍA}

Como se mencionó anteriormente para estimar el exceso de choques es necesario obtener la función de desempeño de seguridad vial para la población de referencia. La estimación de dicha función de desempeño parte de la distribución estadística de los datos supuesta. Dado que los datos de frecuencia de choques corresponden a recuentos y las frecuencias son normalmente bajas, la distribución de Poisson es un buen punto de inicio (Jovanis y Chang, 1986).

La función de probabilidad para la distribución de Poisson viene dada por la ecuación 1.

$$
1 \quad \operatorname{Pr}(z)=\frac{e^{\lambda} \lambda^{z}}{z !}, \quad z=0,1, \ldots
$$

donde $\operatorname{Pr}(\mathrm{z})$ es la probabilidad de observar z número de choques y $\lambda$ es el número esperado de choques.

Ahora el logaritmo natural del número esperado de choques puede ser modelado como una combinación lineal de variables independientes:
2

$$
\ln \lambda_{i}=X_{i} \beta
$$

donde $\mathrm{X}$ es un vector de variables independientes correspondiente a la observación i-ésima y $\beta$ es el vector de coeficientes correspondientes.

Ahora bien, se conoce que la frecuencia de choques viales es normalmente sobredispersa: los accidentes presentan más dispersión de la que una distribución de Poisson puede explicar. Esto porque la varianza en dicha distribución está restringida a ser igual que el promedio (Shankar et al., 1995). Por lo anterior es común que se utilice la distribución binomial negativa que se basa en la distribución de Poisson pero permite que la varianza sea mayor que el promedio (Shankar et al., 1995: AgüeroValverde y Jovanis, 2006).

Una distribución binomial negativa puede a ser considerada como distribución de Poisson con promedio $\lambda$, que a su vez es una variable aleatoria con distribución Gamma como se describe:

3

$$
\ln \lambda_{i}=X_{i} \beta+\varepsilon_{i}
$$

donde $\varepsilon$ es el error:

4

$$
e^{\varepsilon} \sim \operatorname{Gamma}(\phi, \phi)
$$

Aquí $\varepsilon$ controla la cantidad de variación extra-Poisson debido a la heterogeneidad en los datos de choques. Esta parametrización de la distribución Gamma tiene una media de uno y una varianza igual a $1 /$. El parámetro es también conocido el parámetro de sobredispersión.

La distribución de probabilidad marginal es entonces:

$$
\begin{gathered}
\operatorname{Pr}(z)=\int_{0}^{\infty} \frac{e^{-\lambda} \lambda^{z}}{z !} \frac{\phi^{\phi} \lambda^{\phi-1} e^{-\phi \lambda}}{\Gamma(\phi)} \mathrm{d} \lambda= \\
\frac{\Gamma(z+\phi)}{z ! \Gamma(\phi)}\left(\frac{\phi}{\phi+1}\right)^{\phi}\left(1-\frac{\phi}{\phi+1}\right)^{z}, \mathrm{z}=0,1, \ldots
\end{gathered}
$$

donde $\Gamma(\bullet)$ es la función Gamma.

La función de desempeño es estimada usando un modelo generalizado lineal con una distribución de probabilidad binomial negativa. La tasa de choques de la distribución es estimada como una función de la exposición como se muestra en la ecuación 6:

\section{$6 \ln \left(\lambda_{i}\right)=\beta_{0}+\beta_{a} \ln \left(V K M_{i}\right)+\varepsilon_{i}$}

donde $\beta_{0}$ es el término correspondiente la constante, $\beta_{\mathrm{a}}$ es el coeficiente correspondiente a la exposición observada en cada ruta (en unidades de vehículo-kilómetro; VKM). 
Una vez estimados los coeficientes, el número predicho de choques es calculado por medio de la función de desempeño de seguridad, la cual es:

7

$$
N_{\text {pred }}=e^{\beta_{0}} * V K M^{\beta_{a}}
$$

Seguidamente, se calcula lo que se conoce como ajuste por peso, que se refiere a la confiabilidad de la función de desempeño de seguridad que se está utilizando. Si el parámetro de sobredispersiónes es bajo, lo cual indica una menor dispersión y mayor confiabilidad, se tendrá un ajuste por peso más grande, que a su vez indica que la función de desempeño es más confiable. El peso se calcula de la siguiente manera:

8

$$
w=\frac{1}{1+\phi\left(N_{\text {pred }}\right)}
$$

en donde $\varnothing$ es el parámetro de sobre-dispersión de la función de desempeño de seguridad. En este caso en específico, $w$ se conoce como el peso de Bayes empírico.

Finalmente la frecuencia de choques esperados total se calcula por medio de la siguiente ecuación:
$9 \quad N_{e s p}=w * N_{p r e d}+(1-w) * N_{o b s}$

En la cual la variable de $\mathrm{N}_{\text {obs }}$ corresponde al número de choques observados, es decir, los choques que se tienen reportados por parte de los oficiales de tránsito y que fueron recopilados anteriormente.

El paso siguiente consiste en calcular el exceso de frecuencia de choques esperado, que corresponde a la diferencia entre los estimados predichos y los estimados con el ajuste de Bayes empírico:

10

$$
\Delta=N_{e s p}-N_{p r e d}
$$

Dicho exceso permite identificar cuáles rutas tienen una mayor frecuencia de choques esperada que predicha y se espera van a responder mejor a las medidas de mitigación que se propongan, puesto que tienen un exceso importante que puede disminuirse. La Figura 1 muestra de forma gráfica las magnitudes señaladas en las ecuaciones 7 a 10 .

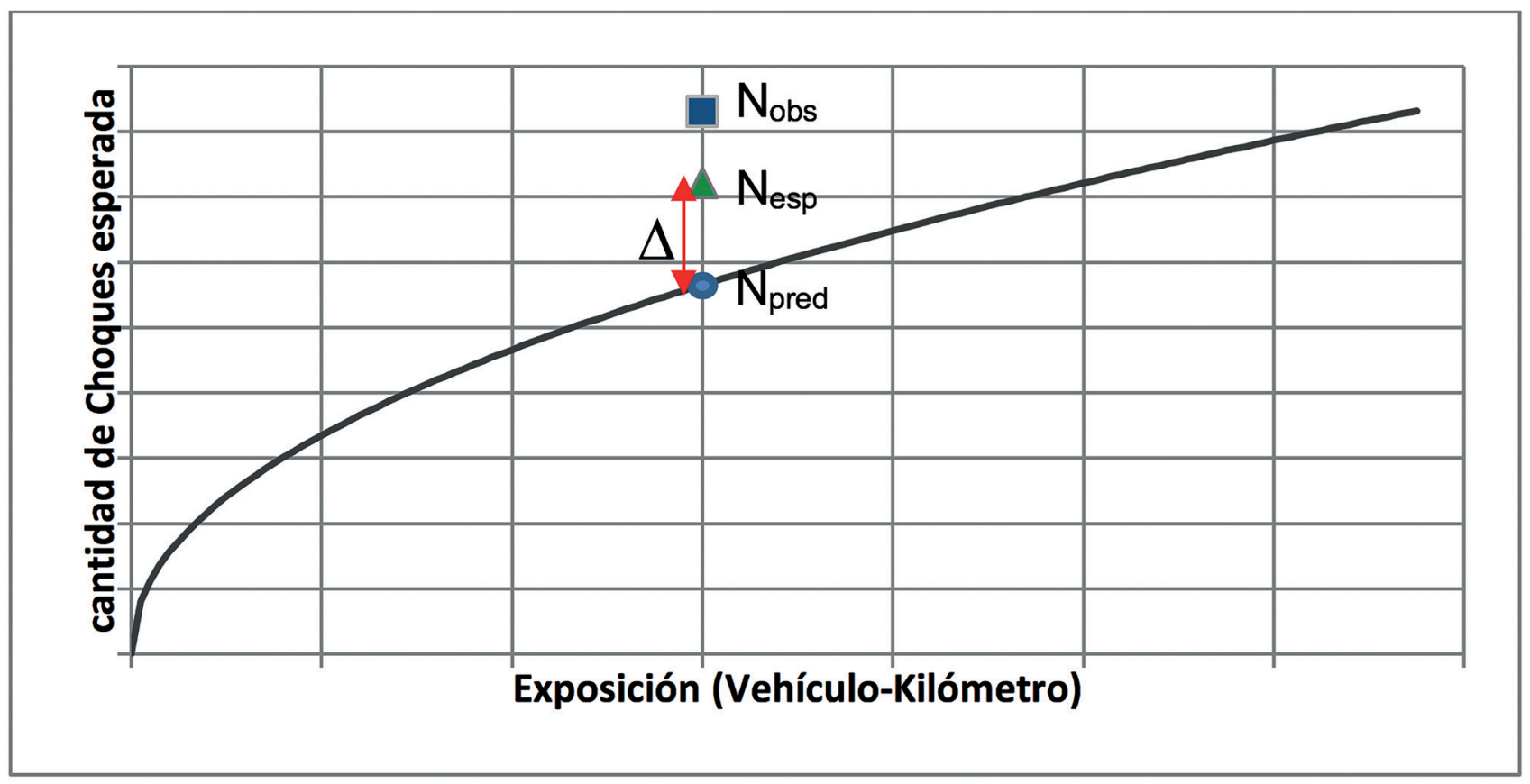

Figura 1. Función de Desempeño de Seguridad Vial 


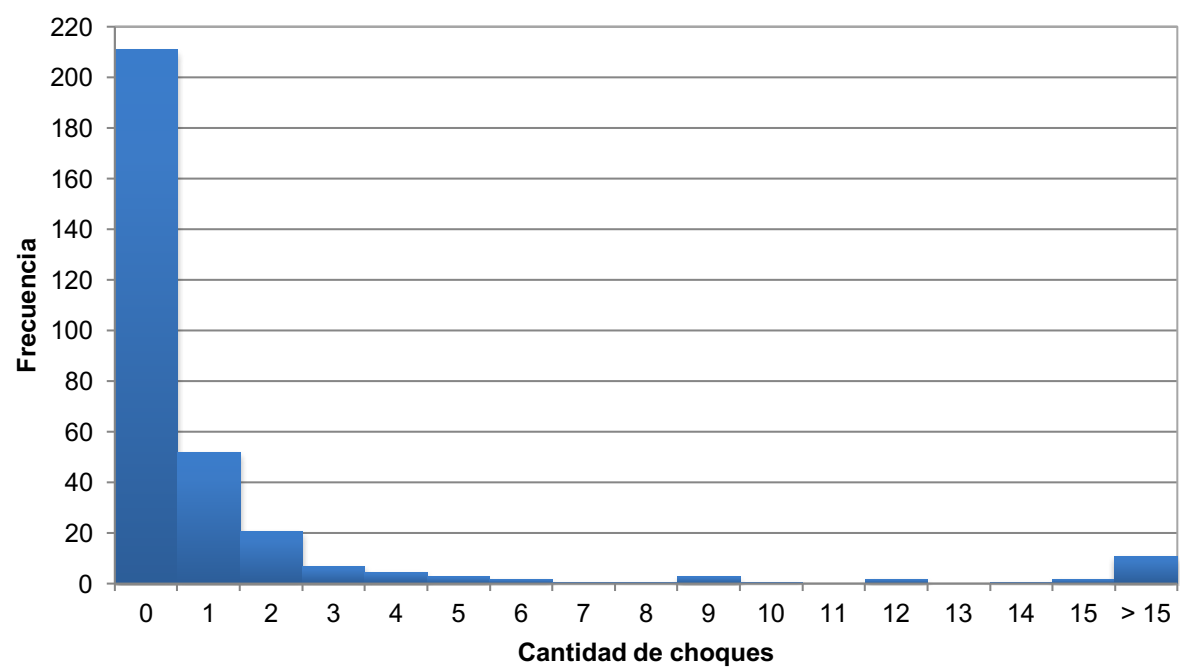

Figura 2. Frecuencia de Choques por ruta años 2010 a 2013.

Fuente: Elaboración propia con datos de Cosevi, 2015.

\section{DESCRIPCIÓN DE LOS DATOS}

\section{Datos de los choques}

Los datos corresponden a la cantidad de muertos en carretera entre los años 2010 a 2013 y fueron suministrados por el Consejo de Seguridad Vial del Ministerio de Obras Públicas y Transportes de Costa Rica (MOPT) (COSEVI, 2015). La red vial nacional reporta 323 rutas de las cuales 211 no experimentaron choques con muertos en sitio durante los cuatro años de análisis, lo cual equivale a un $65 \%$ de las rutas nacionales. Otras 52 rutas presentaron un muerto durante el periodo de análisis mientras que 21 rutas presentaron dos muertos y siete rutas presentaron tres muertos. En resumen, más del 90 \% de las rutas nacionales presentaron menos de cuatro muertos o menos de un muerto al año. La Figura 2 muestra el histograma de frecuencias para la cantidad acumulada de choques por ruta entre los años 2010 y 2014.

El Cuadro 1 presenta la cantidad de muertos en carretera por ruta para el $10 \%$ superior de las rutas. Del cuadro es evidente que las rutas con mayor cantidad de muertos son la Ruta 1, la Ruta 2 y la Ruta 32, todas con más de 100 fallecidos en los cuatro años de estudio. En un distante cuarto lugar se encuentra la ruta 34 con 54 muertos y el quinto lugar lo ocupa la ruta 27 con 53 fallecidos.

Las primeras 33 rutas, que corresponden al $10 \%$ superior de total de muertes en carretera acumulando más del $68 \%$ de la muertes. El otro $90 \%$ de las rutas nacionales acumulan solo el 9,3\% de los fallecidos. Las rutas cantonales por su parte reportan solo el $22.5 \%$ de las muertes en carretera a pesar de que la red cantonal incluía más de 35085 km de vías en el 2014, comparados con los 7232 km de carreteras nacionales (MOPT, 2014)

\section{Datos de las rutas}

La información base de la Red Vial Nacional proviene del Anuario de Información de Tránsito de la Secretaría de Planificación Sectorial también del MOPT (MOPT, 2012; 2013). El anuario presenta el tránsito promedio diario (TPD) estimado para el año, además del porcentaje de crecimiento estimado para los últimos años. Para el caso de los años 2012 y 2013 se utilizó el TPD reportado en el respectivo anuario. Para los años 2010 y 2011 se realizó la respectiva proyección a partir del TPD reportado del 2012 y la tasa de crecimiento en ese mismo año. Tanto el inventario de la Red Vial Nacional como el Anuario tienen información detallada para cada sección de control de la red, dicha información fue agregada por ruta para estimar la exposición en vehículo kilómetro por cada ruta. La Figura 3 muestra el TPD estimado para cada sección de la Red Vial Nacional para el 2013. De la figura es claro que las rutas con mayores volúmenes se concentran en el Área Metropolitana de San José y que una gran cantidad de carreteras nacionales tienen volúmenes menores a los 2800 vehículos diarios.

El Cuadro 2 presenta el acumulado de vehículo-kilómetro por ruta, mostrando el $10 \%$ de las rutas que acumulan mayor exposición. La Ruta 1 exhibe el mayor número de vehículokilómetro con un total de los cuatro años que sobrepasa los 6,3 mil millones de VKM y corresponde al casi $15 \%$ del total de las carreteras nacionales. En un lejano segundo puesto se encuentra la Ruta 2 con un valor de 3,5 mil millones de VKM o poco más de la mitad de exposición de la Ruta 1. La Ruta 27 ocupa el tercer puesto en exposición en Costa Rica con un valor de 2,9 mil millones de VKM. Finalmente, las rutas 32 y 34 completan las 
cinco rutas más altas con un 4\% y un 3,6 \% del total de exposición en VKM de las rutas nacionales respectivamente. Los valores de exposición decaen rápidamente con la Ruta 219 que ocupa el puesto 33 representando solo un 0,54\% del total de VKM. A diferencia de lo que muestra el Cuadro 1, la cantidad de viajes no está tan acumulada en el $10 \%$ mayor de rutas como lo está la cantidad de muertos en sitio; el $10 \%$ mayor de rutas concentra solo el 73 \% de la exposición pero más de un $87 \%$ de las muertes ocurridas en carreteras nacionales. Esto es un claro indicio de que existen rutas que presentan una cantidad de choques mayor de lo que se esperaría dado su nivel de exposición.
Finalmente, el Cuadro 3 presenta las estadísticas descriptivas de las variables de interés. De los datos es evidente la gran dispersión en los valores que existen tanto en la exposición como en la cantidad de muertos en sitio. El promedio para la cantidad de VKM es de apenas 1,39 (x108) mientras que la desviación estándar es de 5,04 (x108). Por su parte la cantidad de muertos en sitio tiene un promedio de 2,88 por ruta pero una desviación estándar de más de 12,52; esto equivale a una varianza de más de 156 lo cual claramente indica variación extra-Poisson en los datos y la necesidad de utilizar un modelo binomial negativo.

Cuadro 1. Cantidad de muertos en carretera por ruta para el $10 \%$ más alto.

\begin{tabular}{|c|c|c|c|c|c|c|}
\hline \multirow{2}{*}{ Ruta Nacional } & \multicolumn{5}{|c|}{ Año } & \multirow{2}{*}{$\begin{array}{l}\text { Porcentaje de } \\
\text { fallecidos }\end{array}$} \\
\hline & 2010 & 2011 & 2012 & 2013 & $2010-13$ & \\
\hline 1 & 30 & 33 & 29 & 26 & 118 & $9,8 \%$ \\
\hline 2 & 34 & 31 & 25 & 25 & 115 & $9,6 \%$ \\
\hline 32 & 22 & 22 & 37 & 25 & 106 & $8,8 \%$ \\
\hline 34 & 20 & 8 & 12 & 14 & 54 & $4,5 \%$ \\
\hline 27 & 11 & 10 & 18 & 14 & 53 & $4,4 \%$ \\
\hline 21 & 7 & 11 & 12 & 17 & 47 & $3,9 \%$ \\
\hline 4 & 16 & 12 & 8 & 10 & 46 & $3,8 \%$ \\
\hline 35 & 8 & 9 & 9 & 10 & 36 & $3,0 \%$ \\
\hline 39 & 5 & 9 & 8 & 7 & 29 & $2,4 \%$ \\
\hline 36 & 2 & 6 & 6 & 8 & 22 & $1,8 \%$ \\
\hline 3 & 6 & 4 & 6 & 5 & 21 & $1,8 \%$ \\
\hline 6 & 2 & 5 & 6 & 2 & 15 & $1,3 \%$ \\
\hline 10 & 7 & 2 & 6 & 0 & 15 & $1,3 \%$ \\
\hline 247 & 0 & 3 & 8 & 3 & 14 & $1,2 \%$ \\
\hline 17 & 5 & 1 & 3 & 3 & 12 & $1,0 \%$ \\
\hline 141 & 1 & 3 & 5 & 3 & 12 & $1,0 \%$ \\
\hline 18 & 0 & 2 & 3 & 5 & 10 & $0,8 \%$ \\
\hline 104 & 2 & 6 & 0 & 1 & 9 & $0,8 \%$ \\
\hline 140 & 1 & 4 & 2 & 2 & 9 & $0,8 \%$ \\
\hline 248 & 4 & 1 & 1 & 3 & 9 & $0,8 \%$ \\
\hline 142 & 3 & 2 & 2 & 1 & 8 & $0,7 \%$ \\
\hline 209 & 0 & 2 & 1 & 4 & 7 & $0,6 \%$ \\
\hline 155 & 1 & 3 & 2 & 0 & 6 & $0,5 \%$ \\
\hline 238 & 1 & 2 & 2 & 1 & 6 & $0,5 \%$ \\
\hline 23 & 0 & 0 & 3 & 2 & 5 & $0,4 \%$ \\
\hline 150 & 2 & 1 & 1 & 1 & 5 & $0,4 \%$ \\
\hline 250 & 0 & 1 & 2 & 2 & 5 & $0,4 \%$ \\
\hline 122 & 4 & 0 & 0 & 0 & 4 & $0,3 \%$ \\
\hline
\end{tabular}




\begin{tabular}{|r|r|r|r|r|r|r|}
\hline $\mathbf{1 2 6}$ & 1 & 1 & 1 & 1 & $\mathbf{4}$ & $0,3 \%$ \\
\hline $\mathbf{1 5 4}$ & 0 & 4 & 0 & 0 & $\mathbf{4}$ & $0,3 \%$ \\
\hline $\mathbf{1 6 0}$ & 1 & 0 & 1 & 2 & $\mathbf{4}$ & $0,3 \%$ \\
\hline $\mathbf{7 0 2}$ & 1 & 2 & 0 & 1 & $\mathbf{4}$ & $0,3 \%$ \\
\hline $\begin{array}{l}\text { Suma 10\% } \\
\text { más alto }\end{array}$ & 199 & 200 & 220 & 198 & $\mathbf{8 1 7}$ & $68,2 \%$ \\
\hline $\begin{array}{l}\text { Otras rutas } \\
\text { nacionales }\end{array}$ & 25 & 19 & 38 & 30 & $\mathbf{1 1 2}$ & $9,3 \%$ \\
\hline $\begin{array}{l}\text { Carreteras } \\
\text { Cantonales }\end{array}$ & $\mathbf{7 4}$ & 69 & 66 & 60 & $\mathbf{2 6 9}$ & $22,5 \%$ \\
\hline TOTAL & $\mathbf{2 9 8}$ & $\mathbf{2 8 8}$ & $\mathbf{3 2 4}$ & $\mathbf{2 8 8}$ & $\mathbf{1 1 9 8}$ & $\mathbf{3}$ \\
\hline
\end{tabular}

Fuente: Elaboración propia con datos de Cosevi, 2015.

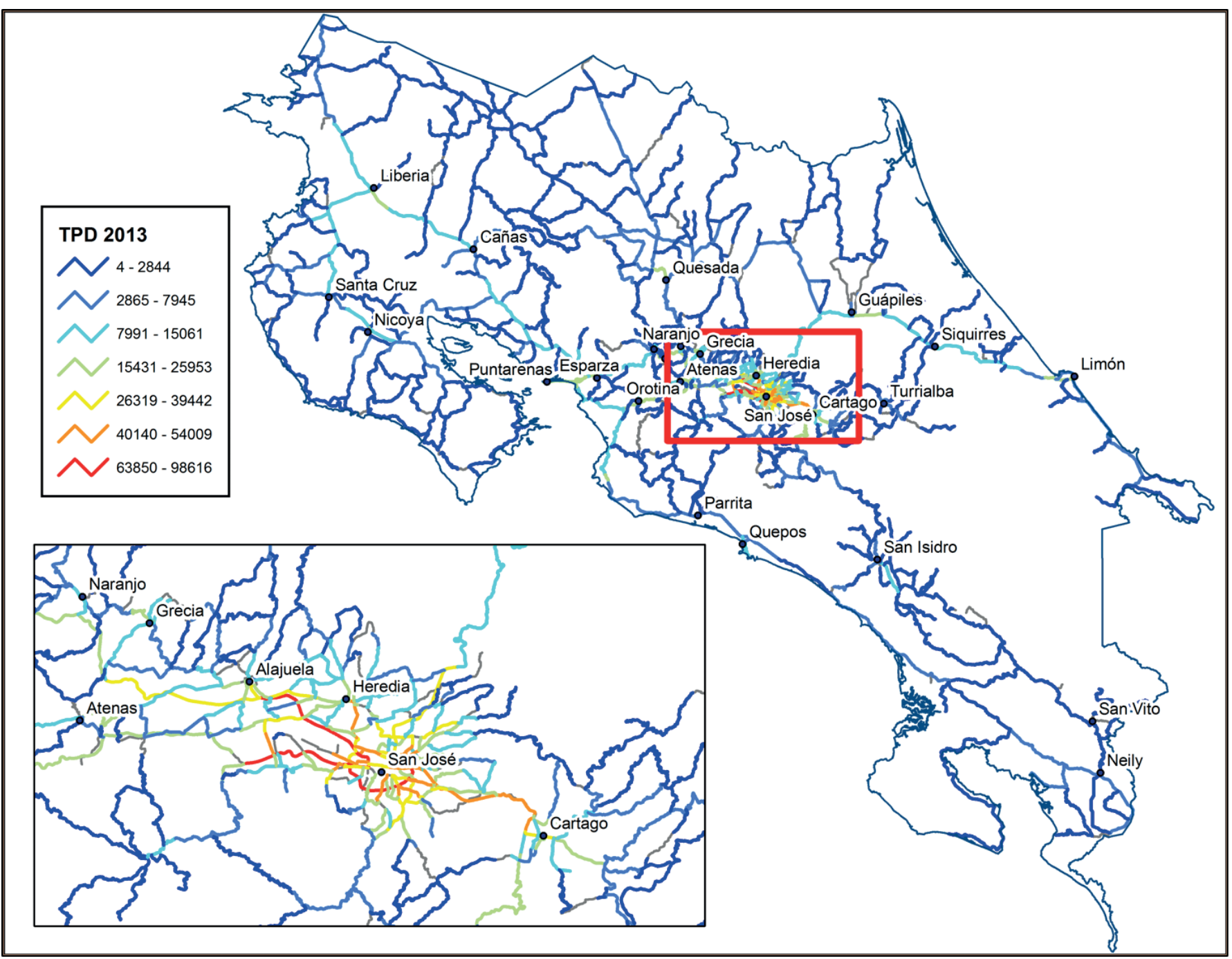

Figura 2. Tránsito Promedio Diario en las secciones de control de la Red Vial Nacional, 2013. 
Cuadro 2. Vehículo-Kilómetro (VKM) por Ruta para el 10\% más alto (100x106)

\begin{tabular}{|c|c|c|c|c|c|c|}
\hline \multirow{2}{*}{ Ruta } & \multicolumn{5}{|c|}{ VKM } & \multirow{2}{*}{ Porcentaje } \\
\hline & 2010 & 2011 & 2012 & 2013 & Total & \\
\hline 1 & 15,02 & 15,71 & 16,00 & 17,19 & 63,92 & $14,84 \%$ \\
\hline 2 & 8,40 & 8,76 & 8,94 & 9,14 & 35,25 & $8,18 \%$ \\
\hline 27 & 6,72 & 7,07 & 7,36 & 7,67 & 28,82 & $6,69 \%$ \\
\hline 32 & 6,67 & 6,90 & 7,09 & 7,32 & 27,99 & $6,50 \%$ \\
\hline 34 & 3,88 & 4,14 & 4,39 & 4,69 & 17,09 & $3,97 \%$ \\
\hline 3 & 3,37 & 3,95 & 4,00 & 4,05 & 15,38 & $3,57 \%$ \\
\hline 39 & 3,42 & 3,45 & 3,52 & 3,56 & 13,95 & $3,24 \%$ \\
\hline 21 & 3,04 & 3,37 & 3,55 & 3,75 & 13,71 & $3,18 \%$ \\
\hline 141 & 2,05 & 2,11 & 2,17 & 2,23 & 8,56 & $1,99 \%$ \\
\hline 10 & 1,79 & 1,86 & 1,93 & 2,00 & 7,58 & $1,76 \%$ \\
\hline 4 & 1,73 & 1,83 & 1,94 & 2,05 & 7,55 & $1,75 \%$ \\
\hline 209 & 1,43 & 1,47 & 1,50 & 1,53 & 5,93 & $1,38 \%$ \\
\hline 218 & 1,16 & 1,19 & 1,22 & 1,24 & 4,81 & $1,12 \%$ \\
\hline 35 & 1,09 & 1,14 & 1,21 & 1,27 & 4,71 & $1,09 \%$ \\
\hline 17 & 0,95 & 0,97 & 1,00 & 1,02 & 3,93 & $0,91 \%$ \\
\hline 215 & 0,97 & 0,98 & 0,98 & 0,99 & 3,92 & $0,91 \%$ \\
\hline 36 & 0,86 & 0,93 & 0,99 & 1,05 & 3,82 & $0,89 \%$ \\
\hline 142 & 0,82 & 0,86 & 0,90 & 0,94 & 3,52 & $0,82 \%$ \\
\hline 102 & 0,86 & 0,87 & 0,87 & 0,89 & 3,48 & $0,81 \%$ \\
\hline 118 & 0,83 & 0,86 & 0,88 & 0,90 & 3,47 & $0,80 \%$ \\
\hline 126 & 0,80 & 0,83 & 0,87 & 0,91 & 3,40 & $0,79 \%$ \\
\hline 111 & 0,84 & 0,84 & 0,85 & 0,85 & 3,38 & $0,78 \%$ \\
\hline 140 & 0,78 & 0,82 & 0,85 & 0,89 & 3,34 & $0,78 \%$ \\
\hline 108 & 0,81 & 0,82 & 0,81 & 0,82 & 3,26 & $0,76 \%$ \\
\hline 18 & 0,67 & 0,71 & 0,80 & 0,86 & 3,04 & $0,71 \%$ \\
\hline 105 & 0,73 & 0,74 & 0,75 & 0,76 & 2,97 & $0,69 \%$ \\
\hline 104 & 0,68 & 0,69 & 0,71 & 0,72 & 2,81 & $0,65 \%$ \\
\hline 121 & 0,60 & 0,68 & 0,70 & 0,71 & 2,69 & $0,62 \%$ \\
\hline 23 & 0,58 & 0,61 & 0,64 & 0,68 & 2,52 & $0,58 \%$ \\
\hline 202 & 0,57 & 0,63 & 0,65 & 0,66 & 2,51 & $0,58 \%$ \\
\hline 147 & 0,60 & 0,62 & 0,64 & 0,65 & 2,51 & $0,58 \%$ \\
\hline 160 & 0,55 & 0,61 & 0,65 & 0,69 & 2,50 & $0,58 \%$ \\
\hline 219 & 0,55 & 0,57 & 0,58 & 0,60 & 2,31 & $0,54 \%$ \\
\hline $\begin{array}{c}\text { Suma } 10 \% \\
\text { Superior }\end{array}$ & 73,82 & 77,57 & 79,95 & 83,29 & 314,63 & $73,03 \%$ \\
\hline $\begin{array}{l}\text { Otras Rutas } \\
\text { nacionales }\end{array}$ & 27,15 & 28,55 & 29,70 & 30,80 & 116,20 & $26,97 \%$ \\
\hline TOTAL & 100,97 & 106,13 & 109,65 & 114,09 & 430,83 & \\
\hline
\end{tabular}


Cuadro 3. Estadísticas descriptivas de las variables por ruta

\begin{tabular}{|c|c|c|c|}
\hline & VKM* & $\begin{array}{c}\text { Muertos en } \\
\text { Sitio }\end{array}$ & Longitud (km) \\
\hline Promedio & 1,39 & 2,88 & 22,39 \\
\hline desviación estándar & 5,04 & 12,52 & 36,58 \\
\hline percentil 25 & 0,05 & 0 & 6,26 \\
\hline Media & 0,18 & 0 & 12,37 \\
\hline Percentil 75 & 0,88 & 1 & 21,69 \\
\hline Max & 63,92 & 118 & 349,40 \\
\hline Mín & 0,002 & 0 & 0,374 \\
\hline
\end{tabular}

*Vehículo-kilómetro (100x106)

\section{RESULTADOS}

\section{Funciones de desempeño de seguridad vial}

Los resultados del Modelo Binomial Negativo para la estimación de la función de desempeño de seguridad para las rutas nacionales de Costa Rica se muestran en el Cuadro 4. El modelo es altamente significativo como lo muestran tanto las probabilidades de los coeficientes como el pseudo $\mathrm{R}^{2}$ que alcanza un $70 \%$. La sobredispersión del modelo es altamente significativa, como lo muestra el valor de $\mathrm{k}$, por lo que la necesidad de utilizar un modelo Binomial Negativo es evidente. Por otro lado, el coeficiente para la exposición ( $\mathrm{\beta a}$ ) es ligeramente menor a uno, lo que implica que el valor esperado de choques aumenta con los Vehículo-kilómetro (VKM) pero a una tasa decreciente. Este resultado es consistente con estudios previos y claramente muestra que la utilización de tasas de choques por VKM no es adecuada pues la relación entre las variables no es completamente lineal (el coeficiente o potencia es diferente de 1 ).

La Figura 3, por su parte, muestra la función de desempeño de seguridad vial estimada para los choques fatales en las rutas nacionales de Costa Rica, así como los valores esperados de muertes en carretera para cada ruta. El número de ruta se presenta para las 10 rutas con mayor exceso de muertes como se detallará en la siguiente sección.

Cuadro 4. Modelo Binomial negativo para la Función de Desempeño de Seguridad Vial de Muertos en

Sitio para las Rutas Nacionales de Costa Rica

\begin{tabular}{|c|c|c|c|c|}
\hline Coeficientes & Estimado & Error Estándar & Valor de z & $\operatorname{Pr}(>|z|)$ \\
\hline Constante $\left(\boldsymbol{\beta}_{0}\right)$ & 0,624 & 0,101 & 6,15 & $7,78 \mathrm{E}-10$ \\
\hline $\operatorname{Ln}(V K M)\left(\beta_{a}\right)$ & 0,931 & 0,064 & 14,63 & $2,00 \mathrm{E}-16$ \\
\hline$k(k=1 / \varnothing)$ & 0,751 & 0,141 & 5,33 & $5,01 \mathrm{E}-08$ \\
\hline Devianza Nula: & 786,36 & \multicolumn{2}{|c|}{ Grados de libertad } & 322 \\
\hline Devianza Residual: & 236,62 & \multicolumn{2}{|c|}{ Grados de libertad } & 321 \\
\hline AIC: & 761,23 & & & \\
\hline 2*In(máxima verosimilitud): & $-755,23$ & & & \\
\hline McFaddenPseudo $\mathrm{R}^{2}$ & 0,70 & & & \\
\hline
\end{tabular}




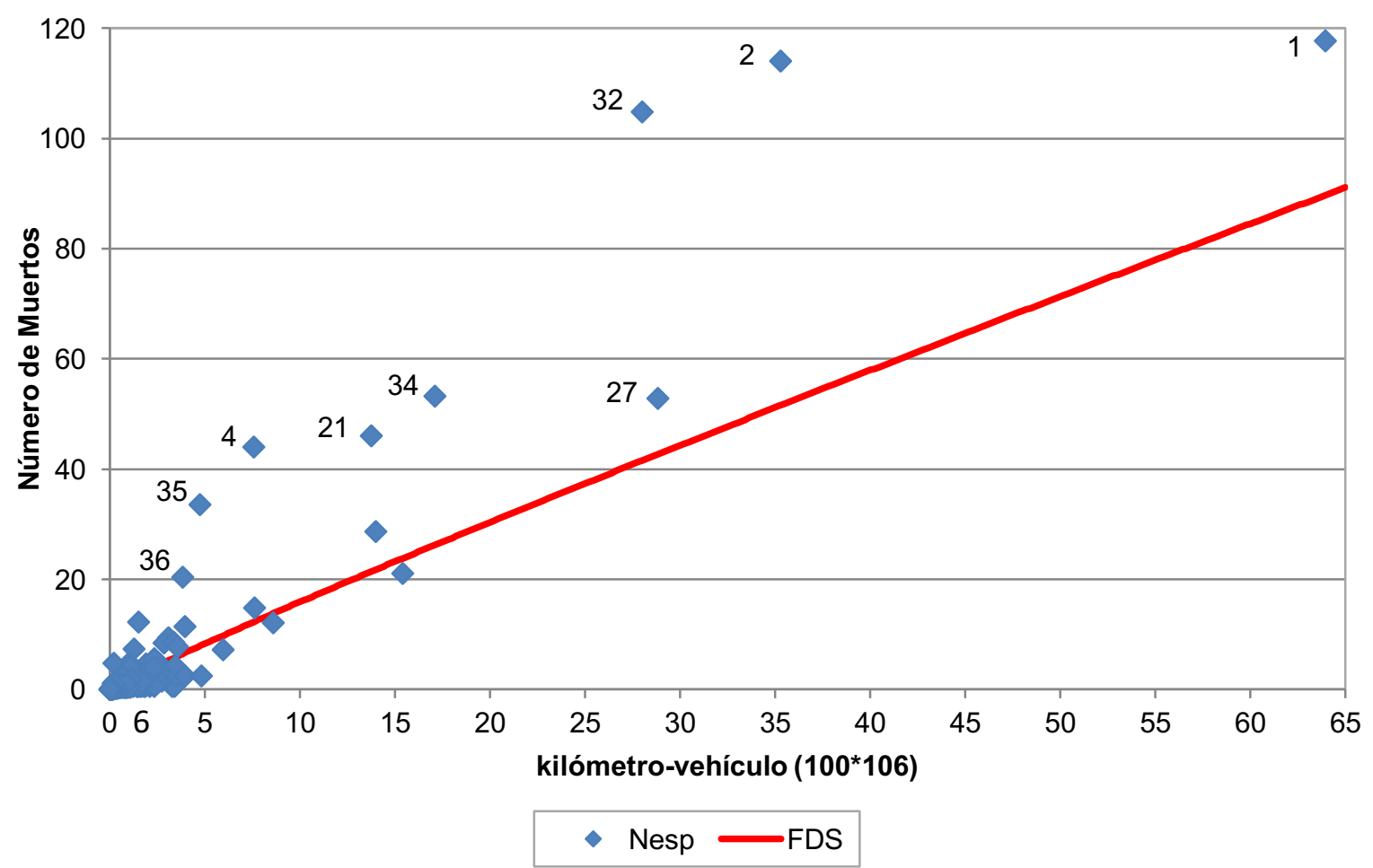

Figura 3. Función de Desempeño de Seguridad Vial para las rutas nacionales de Costa Rica.

\section{Exceso de frecuencia de muertes en carretera con ajuste de Bayes empírico}

El Cuadro 5 presenta las 33 rutas (10\% más alto) que presentan mayor exceso de frecuencia de muertes esperado $(\Delta)$. El mapa de la Figura 4 presenta además el exceso de frecuencia de muertes esperado para cada ruta nacional.

Los resultados del modelo indican que la ruta más peligrosa de Costa Rica es la Ruta 32 con un exceso de muertes de más de 63 durante los cuatro años de análisis, seguida de la Ruta 2 con un exceso de más de 62. En el tercer lugar la Ruta 4 con un delta de 32; le sigue en el cuarto lugar la Ruta 1 con un exceso de más de 28 y completa los primeros cinco lugares la Ruta 34 con un delta de 27. Las otras 5 rutas que completan las 10 más peligrosas son en orden la Ruta 35, la Ruta 21, la Ruta 36, la Ruta 27 y la Ruta 6. La Figura 4 muestra la localización de las 10 rutas con mayor exceso de muertes en carretera.
La Figura 3 muestra que hay rutas por encima y por debajo de la función de desempeño, lo que implica deltas positivos y negativos respectivamente. El mapa de la Figura 4 muestra esta situación más claramente con tres categorías de delta negativas, una cercana al cero y tres positivas. Si se acumula el exceso de muertes en carretera para todas las rutas que tienen un delta positivo se obtiene un valor de 356 muertes en los cuatro años o aproximadamente 90 por año. Las primeras 10 rutas acumulan 296 de esas muertes o lo que es equivalente a más de un $83 \%$ del exceso. Lo anterior evidencia la importancia de concentrar esfuerzos en estas rutas para reducir sustancialmente el número de muertes en carretera en Costa Rica. 
Cuadro 5. Exceso de frecuencia de muertes esperada por ruta para el 10\% más alto

\begin{tabular}{|c|c|c|c|c|c|c|}
\hline Orden & ruta & VKM* & $\mathbf{N}_{\text {obs }}$ & $\mathbb{N}_{\text {pred }}$ & $N_{e s p}$ & $\begin{array}{c}\text { Exceso de } \\
\text { Choques DELTA } \\
(\Delta)\end{array}$ \\
\hline 1 & 32 & 27,99 & 106 & 41,54 & 104,86 & 63,31 \\
\hline 2 & 2 & 35,25 & 115 & 51,50 & 114,09 & 62,58 \\
\hline 3 & 4 & 7,55 & 46 & 12,26 & 44,05 & 31,80 \\
\hline 4 & 1 & 63,92 & 118 & 89,66 & 117,76 & 28,10 \\
\hline 5 & 34 & 17,09 & 54 & 26,24 & 53,23 & 26,98 \\
\hline 6 & 35 & 4,71 & 36 & 7,90 & 33,56 & 25,66 \\
\hline 7 & 21 & 13,71 & 47 & 21,37 & 46,13 & 24,76 \\
\hline 8 & 36 & 3,82 & 22 & 6,50 & 20,40 & 13,89 \\
\hline 9 & 27 & 28,82 & 53 & 42,70 & 52,82 & 10,13 \\
\hline 10 & 6 & 1,48 & 15 & 2,69 & 12,32 & 9,62 \\
\hline 11 & 39 & 13,95 & 29 & 21,72 & 28,76 & 7,03 \\
\hline 12 & 248 & 1,25 & 9 & 2,29 & 7,34 & 5,05 \\
\hline 13 & 17 & 3,93 & 12 & 6,68 & 11,46 & 4,78 \\
\hline 14 & 247 & 0,17 & 14 & 0,36 & 4,78 & 4,42 \\
\hline 15 & 18 & 3,04 & 10 & 5,26 & 9,41 & 4,15 \\
\hline 16 & 104 & 2,81 & 9 & 4,88 & 8,45 & 3,57 \\
\hline 17 & 238 & 0,92 & 6 & 1,72 & 4,70 & 2,98 \\
\hline 18 & 140 & 3,34 & 9 & 5,74 & 8,62 & 2,88 \\
\hline 19 & 10 & 7,58 & 15 & 12,30 & 14,84 & 2,54 \\
\hline 20 & 250 & 1,03 & 5 & 1,92 & 4,13 & 2,21 \\
\hline 21 & 142 & 3,52 & 8 & 6,03 & 7,78 & 1,75 \\
\hline 22 & 155 & 2,30 & 6 & 4,05 & 5,70 & 1,64 \\
\hline 23 & 150 & 1,88 & 5 & 3,36 & 4,70 & 1,34 \\
\hline 24 & 154 & 1,35 & 4 & 2,47 & 3,64 & 1,18 \\
\hline 25 & 249 & 0,49 & 3 & 0,96 & 2,10 & 1,14 \\
\hline 26 & 164 & 0,55 & 3 & 1,07 & 2,21 & 1,13 \\
\hline 27 & 415 & 0,66 & 3 & 1,27 & 2,36 & 1,09 \\
\hline 28 & 223 & 0,24 & 3 & 0,49 & 1,48 & 0,99 \\
\hline 29 & 713 & 0,16 & 3 & 0,34 & 1,17 & 0,83 \\
\hline 30 & 244 & 0,30 & 2 & 0,61 & 1,23 & 0,62 \\
\hline 31 & 152 & 0,41 & 2 & 0,80 & 1,42 & 0,62 \\
\hline 32 & 933 & 0,22 & 2 & 0,45 & 1,03 & 0,58 \\
\hline 33 & 165 & 0,18 & 2 & 0,37 & 0,91 & 0,54 \\
\hline
\end{tabular}

*VKM: Vehículo-kilómetro (100×106) 


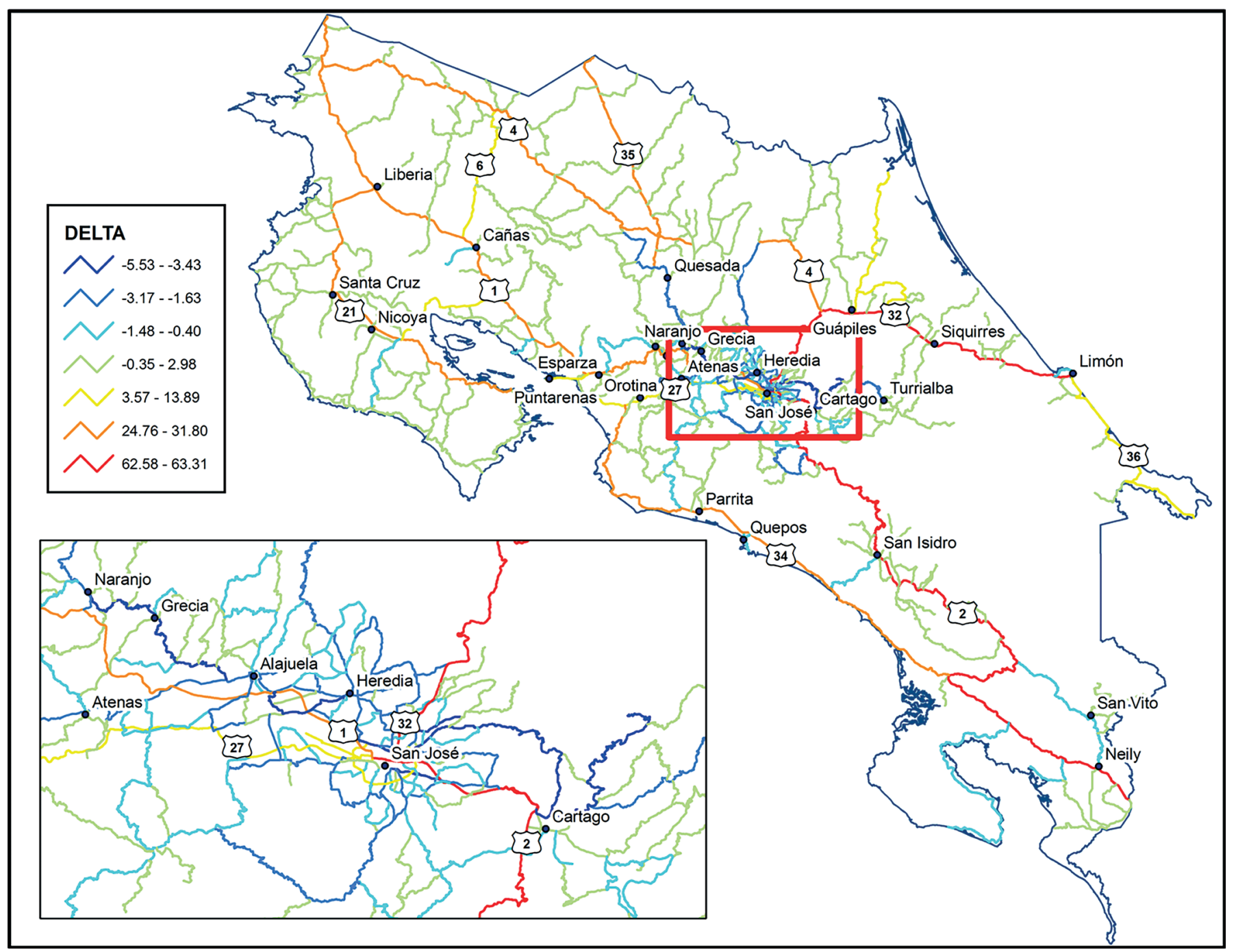

Figura 4. Exceso de frecuencia de muertes esperada por ruta

Las 10 primeras rutas por exceso de muertes son esencialmente rurales aunque algunas como las rutas 32, 1, 2 y 27 presentan segmentos cortos de autopistas urbanas que concentran una gran cantidad de los vehículos-kilómetro de la Gran Área Metropolitana de San José. Lo anterior muestra indicios de que los viajes rurales conllevan mayor riesgo que los viajes urbanos en Costa Rica.

El cálculo de exceso de muertes en carretera ofrece la ventaja analítica de presentar de forma directa las rutas que estadísticamente presentan la mayor probabilidad de reducción de choques. Esto por cuanto el delta representa la diferencia entre el valor esperado para cada ruta y el valor predicho para rutas similares en condiciones normales; o dicho de otra forma el delta mide cuanto se aleja la ruta de la normalidad. Estadísticamente hablando, podría ser más factible reducir 20 muertes en la ruta 32 que presenta un exceso de más de 63 que reducir 20 muertes en la ruta 6 que presenta apenas 9 muertes en exceso.
En este punto se aclara que todas las muertes por choques viales son prevenibles y por lo tanto deberíamos hacer un esfuerzo por reducirlas a cero en todo el país. Sin embargo, los recursos son escasos por lo que concentrar esfuerzos en las rutas con mayor exceso de muertes producirá las mayores reducciones con los recursos disponibles.

Los usuarios de las rutas con mayor exceso de muertes en carretera están sujetos a un riesgo vial desproporcionalmente mayor que de los usuarios del resto de las vías y es nuestra responsabilidad hacer lo posible para lograr una reducción de ese riesgo. La identificación de las rutas con mayor exceso de muertes es un primer paso para lograr dicha reducción de riesgo. A éste debe seguir un análisis detallado de cada una de las rutas con exceso de muertes para realizar auditorías de seguridad vial, determinar los tramos de concentración de choques y las medidas de mitigación correspondientes. 


\section{CONCLUSIONES}

Los resultados del modelo indican que la ruta más peligrosa de Costa Rica en términos de exceso de muertes en carretera es la Ruta 32, seguida de cerca por la Ruta 2. Lejos de estas dos se encuentra en el tercer lugar la Ruta 4, en cuarto lugar la Ruta 1 y en quinto lugar la Ruta 34. Las otras cinco rutas que completan las 10 más peligrosas son en orden la Ruta 35, la Ruta 21, la Ruta 36, la Ruta 27 y la Ruta 6.

El análisis realizado demuestra que el método de exceso de choques esperado con el ajuste de Bayes empírico usando funciones de desempeño de seguridad vial es apropiado para realizar un análisis de concentración de muertes por ruta. El estudio permitió identificar las rutas más peligrosas de Costa Rica según el exceso de muertes en carretera. Las rutas identificadas como las más peligrosas deberían ser objeto de un estudio de tramos de concentración de choques y una auditoría de seguridad vial para cada tramo identificado.

Las primeras 10 rutas por exceso de muertes en carretera acumulan un $83 \%$ del total de exceso. Lo anterior demuestra que si nos concentráramos en estas 10 rutas podría lograrse una reducción substancial de muertes por choques viales en Costa Rica.

La estimación del exceso de muertes en carretera ofrece la ventaja de ordenar las rutas que estadísticamente presentan la mayor probabilidad de reducción de choques y que por lo tanto ofrecen la mejor relación beneficio-costo para la reducción de choques.

\section{REFERENCIAS}

1. AASHTO.(2010). Highway Safety Manual. American Association of State and Highway Transportation Officials, Washington D.C. USA

2. Aguero-Valverde, J. 2013. Multivariate spatial models of excess crash frequency at area level: Case of Costa Rica. Accident Analysis and Prevention, 59, 365-373.

3. Aguero-Valverde, J., Jovanis, P.P. (2006). Spatial analysis of fatal and injury crashes in Pennsylvania. Accident Analysis and Prevention, 38 (3), 618-625.

4. Barnett, A. G., van der Pols, J. C., \& Dobson, A. J. (2005). Regression to the mean: what it is and how to deal with it. International Journal of Epidemiology, 34(1), 215-220.

5. Castro, E.A., Agüero-Valverde, J. (2015). Aplicación del método Bayes empírico en análisis de seguridad vial: el caso de la ruta 32 en Costa Rica. Revista Ingeniería, 25 (1), pp 13-33.

6. COSEVI (2015). Muertos en sitio por ruta. Área de Investigación y Estadística.

7. Evans, L. (2004). Traffic safety.Science Serving Society.MI, USA

8. Hadayeghi, A., Shalabyb, A.S., Persaud., B.N. (2010). Development of planning level transportation safety tools using Geographically Weighted Poisson Regression. Accident Analysis and Prevention, 42 (2), 676-688.

9. Hakkert, A. S., Braimaister, L., \& Van Schagen, I. (2002). The uses of exposure and risk in road safety studies (Vol. 2002, No. 12). SWOV Institute for Road Safety.

10. Hauer, E. (1992). Empirical Bayes approach to the estimation of "unsafety": the multivariate regression method. Accident Analysis \& Prevention, 24(5), 457-477.

11. Hauer, E. (1995). On exposure and accident rate. Traffic engineering \& control, 36(3), 134-138.

12. Hauer, E. (1997). Observational Before/After Studies in Road Safety.Estimating the Effect of Highway and Traffic Engineering Measures on Road Safety.Emeral, IWA, UK

13. Hauer, E., Harwood, D., Council, F., \& Griffith, M. (2002).Estimating safety by the empirical Bayes method: a tutorial. Transportation Research Record, 1784, 126-131.

14. Huang H., Darwiche, A.L., Abdel-Aty, M.A. (2010). County-Level Crash Risk Analysis in Florida: Bayesian Spatial Modeling. TransportationResearch Record, 2148, 27-37. 
15. INEC (2014). Unidad de Estadísticas Demográficas - Defunciones (2000-2014) http://www.inec.go.cr/bincri/RpWebEngine.exe/ Portal?BASE=VITDEF

16. Jovanis, P. P., \& Chang, H. L. (1986). Modeling the relationship of accidents to miles traveled. Transportation Research Record, $1068,42-51$.

17. MacNab, Y.C. (2004). Bayesian spatial and ecological models for small-area accident and injury analysis.Accident Analysis and Prevention, 36 (6), 1019-1028.

18. Miaou S-P., Lord, D. (2003). Modeling traffic crash-flow relationships for intersections: dispersion parameter, functional form and Bayes versus empirical Bayes.TransportationResearch Record 1840, 31- 40.

19. MOPT (2012). Anuario de Información de Tránsito 2012, Departamento de Planificación Sectorial

20. MOPT (2013). Anuario de Información de Tránsito 2013, Departamento de Planificación Sectorial

21. MOPT (2014). Red Vial Cantonal Longitud Total - Longitud para la Distribución de Fondos Ley 8114 - Resumen Por Cantón 2014 http://mopt.go.cr/carreteras/RVC.pdf

22. Qin, X., Ivan, J. N., \& Ravishanker, N. (2004). Selecting exposure measures in crash rate prediction for two-lane highway segments. Accident Analysis \& Prevention, 36(2), 183-191.

23. Quddus M.A. (2008). Modeling area-wide count outcomes with spatial correlation and heterogeneity: an analysis of London crash data.Accident Analysis and Prevention, 40(4), 1486-1497.

24. Shankar, V., Mannering, F., \& Barfield, W. (1995).Effect of roadway geometrics and environmental factors on rural freeway accident frequencies. Accident Analysis \& Prevention, 27(3), 371-389.

25. Shinar, D. (1978). Psychology on the road.The human factor in traffic safety. John Wiley \& Sons, NJ, USA.

26. Siddiqui, C., Abdel-Aty, M.A., Choi.K., (2012). Macroscopic spatial analysis of pedestrian and bicycle crashes. Accident Analysis and Prevention, 45 (1), 382-391.

27. Toroyan, T. (2013).Global status report on road safety 2015. Supporting a decade of action. Geneva: World Health Organization, Department of Violence and Injury Prevention and Disability.

28. (PIARC) (2003).Road Safety Manual, World Road Association Paris, Francia. 